ORIGINAL ARTICLE

\title{
Assessment of Liver Stiffness among Chronic Hepatitis C Patients after Oral Antiviral Drugs in Zagazig University Hospitals
}

\author{
Mohammed Hisham Mounir ${ }^{1}$, Amany Mohammed Ibrahim ${ }^{1}$, Sameh Saber Bayiomy ${ }^{2}$, Hany \\ Mostafa Omar Mohammed ${ }^{{ }^{*}}$ \\ ${ }^{1}$ Internal Medicine Department, Faculty of Medicine, Zagazig University, Zagazig, Egypt \\ ${ }^{2}$ Diagnostic Radiology Department, Faculty of Medicine, Zagazig University, Zagazig, Egypt
}

\section{*Corresponding auther: Hany Mostafa Omar Mohammed Internal Medicine Department, Faculty of Medicine, Zagazig University, Email: Zagazig, Egypt hanymostafa1986@gmail.com}

Submit Date Revise Date Accept Date

2019-03-24 2019-05-15 2019-05-18

\begin{abstract}
Background: The hepatitis $\mathrm{C}$ virus (HCV) may be an important explanation for hepatic pathology, cirrhosis, and hepatocellular carcinoma. The aim of the study was to assess the liver stiffness before and after oral antiviral medication in chronic viral hepatitis $\mathrm{c}$ patients attending Zagazig University Hospitals. Methods: This study included forty three Egyptian patients with HCV infection diagnosed by HCV RNA real time quantitative PCR whose ages were ranged from 29-71, All patients took anti-viral treatment according to the rules of the committee responsible for hepatitis c virus control in Egypt. Treatment regimens included: Sofosbuvir, daclatasvir and ribavirin (RBV). The patients were collected and followed up in the period between April 2017 and August 2018. , radiological tool liver transient elastography (TE) and laboratory tests APRI, FIB4 test were done for all patients. Results: There were improvements in liver stiffness measurements,APRI,FIB4 test hemoglobin, fasting blood sugar , platelets count, and ALT, AST levels in patients who achieved a 48-week sustained infectious agent response. The failure to achieve improvement within the level of liver stiffness was related to treatment failure. Conclusions: Treatment with sofosbuvir drug regimen makes significant decrease in liver stiffness measurements and fibrosis indices.

Key words: Liver stiffness; Fibroscan; Direct antivirals
\end{abstract}

\section{INTRODUCTION}

H epatitis $\mathrm{C}$ Virus (HCV) may be a major reason for hepatic disease, failure and hepatocellular carcinoma, $14.7 \%$ is the highest percentage in the world acquiring the virus infection in Egypt [1].

The treatment of $\mathrm{HCV}$ aimed at achieving a sustained virological response (SVR), to reduce the liver harm, good cure decrease pathology and death rates. Studies before found that HCV cure diminishes hepatocellular carcinoma risk [2]. Staging of hepatic pathology determines liver illness progression throughout chronic infection.

In 2014, the NS5B RNA-polymerase inhibitor sofosbuvir was first drug used for the $\mathrm{HCV}$ treatment in conjunction with pegylated
Interferon- (Peg- IFN) with or without ribavirin (RBV), yielding high SVR rates [3].

The corner stone of liver fibrosis assessment is a liver histopathologic diagnostic test exploitation; using Ishak or Metavir scores, Non-invasive methods assessing liver pathology can replace liver diagnostic test utterly [4], results were obtained with liver stiffness (LS) measurements obtained from transient elastography (TE) (Fibroscan), which is used in routine clinical follow up were suggested to be another technique to evaluate the fibrosis of liver [5]. Serial liver fibrosis measurements are taken for analysis of liver harm caused by HCV [5]. Transient elastography is a suitable tool for getting continual measurements as a result of it 
eliminates all the hazards of invasive liver diagnostic test technique .In great study, they found that fibroscan has a $91 \%$ specificity and an $87 \%$ sensitivity in comparison to biopsy regarding fibrosis in the liver [6].

Researchers found a decline in hepatic fibrosis after treatment in relation to before the treatment [7].

Noninvasive ways evaluating hepatic pathology induced by HCV were planned over few years recently like the AST to platelet magnitude relation index (APRI) and the fibrosis index based on 4 factors (FIB4) [8]. This research goal is to assess the liver stiffness before and after oral antiviral medication in chronic viral hepatitis $\mathrm{c}$ patients attending Zagazig University Hospitals using noninvasive methods like fibroscan and APRI and FIB4 test. .

Site of study

\section{METHODS}

This study was carried out in Gastroenterology and hepatology unit and Advanced center for liver diseases, Internal Medicine Department, Faculty of medicine, Zagazig University Hospitals between April 2017 and August 2018.

Written informed consent was obtained from all participants and the study was approved by the research ethical committee of Faculty of Medicine, Zagazig University. The work has been carried out in accordance with The Code of Ethics of the World Medical Association (Declaration of Helsinki) for studies involving humans.

Type of the study: prospective cohort study.

Sample size

Out of forty five Egyptian patients with chronic $\mathrm{HCV}$ infection diagnosed by HCV PCR (polymerase chain reaction) and fulfilled the inclusion and exclusion criteria of the study, 43 patients (aged 29-71 years old) continued the follow up period and were enrolled in the study.

\section{Inclusion criteria:}

Treatment naïve or treatment experienced patients with chronic HCV infection proven by HCV PCR. Age 18-75 years, any Body Mass
Index (BMI) (weight in kilograms/squared height in meters) was included. Patients with either liver cirrhosis or not, with or without decompensation was included.

\section{Exclusion criteria:}

Patients with other cause of chronic disease like hepatitis B or HIV, bilharzasias or hemochromatosis or chronic alcoholism or wilson illness. Patients with $\mathrm{HCC}$ or any hepatic focal lesion, intravenous drug abusers were excluded.

LS measurements and fibrosis indices (APRI) and (FIB4) were performed to all the study patients at zero week (baseline) and 48 weeks after end of treatment using fibroscan device (transient elastography) and laboratory analysis like complete blood picture (CBC) and liver function test (LFT) alpha fetoprotein (AFP) lipid profile and fasting blood sugar (FBS) and international normalization ratio( INR) were done. HCV PCR was done before treatment, at end of treatment and 48 weeks after treatment.

\section{Statistical analysis}

All data were analyzed using SPSS 20.0 for windows, MedCalc Statistical Software version 15.8. Continuous variables were expressed as the mean $\pm \mathrm{SD}$, median and range while the categorical variables were expressed as a number (percentage). Continuous variables were checked for normality by using Shapiro Wilk test. One-Way ANOVA, Kruskal-Wallis H (KW), Post-hoc Fisher's, Least Significant Difference test (LSD) tests were used, Pearson product-moment correlation coefficient, Receiver operating characteristic (ROC) curve analysis were used. $\mathrm{p}<0.05$ was considered statistically significant $(\mathrm{S})$.

\section{RESULTS}

There was a marked improvement in liver stiffness measurements; fibrosis indices blood hemoglobin levels, blood sugar levels for fasting, platelets, and liver enzymes ALT, AST in patients who achieved a 48 -week sustained virological response more than non-responders (table 1). There is a significant difference between the baseline and 48 weeks groups as regard FBS, $\mathrm{Hb}, \mathrm{AST}$ and positive PCR (table 2) in naieve patients, There is a significant 
difference between the cirrhotic and noncirrhotic patients regarding FIB scan, APPRI, FIB4, Hb, PLT, AST, Albumin, Total bilirubin, alpha FB and TG (table3).

There is a significant difference regarding FIB scan, APPRI, FIB4, AST, Total bilirubin and alpha FB more than experienced group (table 4). The failure to realize improvement within the level of liver stiffness was related to treatment failure, sustained viral response. APRI, FIB4 measurements, alpha FB, FIB Scan measurements were done (table $5)$.

Table 1. Comparison between responders and non-responders.

\begin{tabular}{|c|c|c|c|c|c|}
\hline \multicolumn{2}{|c|}{ Variable } & Responders $(n=39)$ & Non-Responders $(n=4)$ & $t / \chi^{2}$ & $P$ \\
\hline \multicolumn{2}{|c|}{$\begin{array}{c}\text { Age (years) } \\
\text { Mean } \pm S D\end{array}$} & $49.13 \pm 10.21$ & $42.5 \pm 9.98$ & 1.238 & .223 \\
\hline \multirow[t]{2}{*}{$\operatorname{Sex}$} & \multirow{2}{*}{$\begin{array}{c}\text { Male } \\
\text { Female }\end{array}$} & $19(48.7 \%)$ & $1(25 \%)$ & \multirow[t]{2}{*}{.863} & \multirow[t]{2}{*}{.353} \\
\hline & & $20(51.3 \%)$ & $3(75 \%)$ & & \\
\hline \multicolumn{2}{|c|}{ FIB scan } & $8.01 \pm 2.45$ & $15.19 \pm 3.71$ & 5.344 & .000 \\
\hline \multicolumn{2}{|c|}{$A P R I$} & $.832 \pm .422$ & $2.07 \pm 1.08$ & 4.705 & .000 \\
\hline \multicolumn{2}{|c|}{ FIB4 } & $2.365 \pm 1.04$ & $4.778 \pm 3.26$ & 3.446 & .001 \\
\hline \multicolumn{2}{|c|}{$F B S(m g / d l)$} & $112.08 \pm 35.25$ & $129.75 \pm 48.47$ & .925 & .360 \\
\hline \multicolumn{2}{|c|}{ Hemoglobin $(g / d l)$} & $13.64 \pm .869$ & $11.18 \pm 1.03$ & 5.320 & .000 \\
\hline \multicolumn{2}{|c|}{$T L C$} & $7.64 \pm 1.34$ & $7.45 \pm 1.53$ & .266 & .792 \\
\hline \multicolumn{2}{|c|}{$P L T$} & $151.21 \pm 36.45$ & $88.5 \pm 3.69$ & 3.402 & .002 \\
\hline \multicolumn{2}{|c|}{$A L T(u / d l)$} & $46.85 \pm 18.11$ & $60.75 \pm 11.09$ & 1.497 & .142 \\
\hline \multicolumn{2}{|c|}{$A S T(u l d l)$} & $46.89 \pm 19.24$ & $84.75 \pm 22.16$ & 3.704 & .001 \\
\hline \multicolumn{2}{|c|}{ Albumin(gldl) } & $3.87 \pm .502$ & $3.0 \pm .183$ & 3.393 & .002 \\
\hline \multicolumn{2}{|c|}{$I N R$} & $1.02 \pm .067$ & $1.03 \pm .050$ & .056 & .956 \\
\hline \multicolumn{2}{|c|}{ Total bil.(mg/dl) } & $1.029 \pm .228$ & $2.188 \pm .312$ & 9.362 & .000 \\
\hline \multicolumn{2}{|c|}{ Alpha FB(ng/ml) } & $8.9 \pm 4.102$ & $23.5 \pm 2.082$ & 6.971 & .000 \\
\hline \multicolumn{2}{|c|}{$T C(m g / d l)$} & $182.46 \pm 16.37$ & $203.5 \pm 3.87$ & 2.537 & .015 \\
\hline \multicolumn{2}{|c|}{$T G(m g / d l)$} & $148.87 \pm 14.09$ & $144.0 \pm 19.95$ & .635 & .529 \\
\hline \multicolumn{2}{|c|}{$L D L(m g / d l)$} & $92.72 \pm 16.69$ & $95.75 \pm 7.72$ & .356 & .723 \\
\hline \multicolumn{2}{|c|}{$D M$} & $15(38.5 \%)$ & $2(50 \%)$ & .198 & .656 \\
\hline \multirow{2}{*}{\multicolumn{2}{|c|}{ Child-Pugh }} & $37(94.9 \%)$ & $4(100 \%)$ & \multirow[t]{2}{*}{.400} & \multirow[t]{2}{*}{.527} \\
\hline & & $2(5.1 \%)$ & $0(0 \%)$ & & \\
\hline
\end{tabular}

FIBscan = fibroscan,APRI=alt/platelets ratio index, FIB4=fibrosis index based on 4 parameters,TLC=total leucocytic count,PLT $=$ platelets count,INR=international normalization ratio , alpha $\mathrm{FP}=$ alpha fetoprotein, $\mathrm{TC}=$ total cholesterol, $\mathrm{TG}=$ triglycerides, $\mathrm{LDL}=\mathrm{low} \quad$ density $\quad$ lipoprotein, $\mathrm{DM}=\quad$ diabetes mellitus.ALT=alanine aminotransferase, AST=asprtate aminotransferase. Total bil=total bilirubin 
Table 2. Comparison between pre and post treatment.

\begin{tabular}{|l|l|l|l|l|}
\hline Variable & Pre $(\mathbf{n = 4 3})$ & Post $(\boldsymbol{n}=\mathbf{4 3})$ & $\boldsymbol{t} / \chi^{2}$ & $\boldsymbol{P}$ \\
\hline $\begin{array}{l}\text { Age }(\text { years }) \\
\text { Mean } \pm \text { SD }\end{array}$ & $48.51 \pm 10.26$ & $50.14 \pm 11.54$ & .691 & .491 \\
\hline FIB scan(kpa) & $8.673 \pm 3.293$ & $7.598 \pm 3.026$ & 1.576 & .199 \\
\hline APPRI & $.948 \pm .614$ & $.763 \pm .694$ & 1.303 & .196 \\
\hline FIB4 & $2.589 \pm 1.496$ & $2.229 \pm 1.694$ & 1.045 & .299 \\
\hline FBS(mg/dl) & $113.72 \pm 36.32$ & $97.67 \pm 21.40$ & $\mathbf{2 . 4 9 6}$ & $\mathbf{. 0 1 5}$ \\
\hline Hemoglobi(g/dl) & $13.41 \pm 1.13$ & $10.99 \pm 1.19$ & $\mathbf{9 . 6 5 5}$ & $\mathbf{. 0 0 0}$ \\
\hline TLC & $7.62 \pm 1.34$ & $7.47 \pm 1.35$ & .505 & .615 \\
\hline PLT & $145.37 \pm 39.28$ & $161.84 \pm 38.89$ & 1.953 & .054 \\
\hline ALT(u/dl) & $48.14 \pm 17.95$ & $41.33 \pm 13.91$ & 1.968 & .052 \\
\hline AST(u/dl) & $50.42 \pm 22.22$ & $41.23 \pm 19.88$ & $\mathbf{2 . 0 2 0}$ & $\mathbf{. 0 4 7}$ \\
\hline Albumin(g/dl) & $3.79 \pm .544$ & $3.99 \pm .531$ & 1.830 & .071 \\
\hline INR & $1.023 \pm .065$ & $1.032 \pm .102$ & .506 & .614 \\
\hline Total bil.(mg/dl) & $1.14 \pm .412$ & $1.05 \pm .318$ & 1.069 & .288 \\
\hline Alpha FB(ng/ml) & $10.26 \pm 5.83$ & $8.44 \pm 5.65$ & 1.469 & .145 \\
\hline TC(mg/dl) & $184.42 \pm 16.79$ & $187.77 \pm 17.32$ & .911 & .365 \\
\hline TG(mg/dl) & $148.42 \pm 14.51$ & $147.53 \pm 16.03$ & .268 & .789 \\
\hline LDL(mg/dl) & $93.0 \pm 16.03$ & $92.14 \pm 15.0$ & .257 & .798 \\
\hline Positive PCR & $43(100 \%)$ & $4(9.3 \%)$ & $\mathbf{9 1 . 8 6 1}$ & $\mathbf{. 0 0 0}$ \\
\hline Cirrhosis & $10(23.3 \%)$ & $5(11.6 \%)$ & 2.051 & .152 \\
\hline
\end{tabular}

FIBscan = fibroscan,APRI=alt/platelets ratio index, FIB4=fibrosis index based on 4 parameters,TLC=total leucocytic count,PLT $=$ platelets count,INR=international normalization ratio ,alpha $\mathrm{FP}=$ alpha fetoprotein, $\mathrm{TC}=$ total cholesterol, $\mathrm{TG}=$ triglycerides, $\mathrm{LDL}=\mathrm{low} \quad$ density $\quad$ lipoprotein, $\mathrm{DM}=$ diabetes mellitus.,PCR=polymerase chain reaction.FBS=fasting blood sugar. ALT=alanine aminotransferase, AST=asprtate aminotransferase.total bil=total bilirubin 
Table 3. Comparison between patients regarding cirrhosis.

\begin{tabular}{|c|c|c|c|c|c|}
\hline \multicolumn{2}{|c|}{ Variable } & Cirrhotic $(n=10)$ & Non- Cirrhotic ( $n=33)$ & $t / \chi^{2}$ & $\boldsymbol{P}$ \\
\hline \multicolumn{2}{|c|}{$\begin{array}{l}\text { Age (years) } \\
\text { Mean } \pm S D\end{array}$} & $46.9 \pm 9.04$ & $49.0 \pm 10.68$ & .562 & .577 \\
\hline \multirow[t]{2}{*}{ Sex } & Male & $4(40 \%)$ & $16(48.5 \%)$ & \multirow[t]{2}{*}{.224} & \multirow[t]{2}{*}{.636} \\
\hline & Female & $6(60 \%)$ & $17(51.5 \%)$ & & \\
\hline \multicolumn{2}{|c|}{ FIB $\operatorname{scan}(k p a)$} & $13.71 \pm 2.49$ & $7.15 \pm 1.46$ & 10.447 & .000 \\
\hline \multicolumn{2}{|c|}{$A P P R I$} & $1.64 \pm .885$ & $.737 \pm .280$ & 5.192 & .000 \\
\hline \multicolumn{2}{|c|}{ FIB4 } & $4.169 \pm 2.24$ & $2.11 \pm .71$ & 4.655 & .000 \\
\hline \multicolumn{2}{|c|}{$F B S(m g / d l)$} & $117.9 \pm 38.21$ & $112.45 \pm 36.24$ & .411 & .683 \\
\hline \multicolumn{2}{|c|}{ Hemoglobin $(\mathrm{gm} / \mathrm{dl})$} & $12.48 \pm 1.46$ & $13.69 \pm .857$ & 3.289 & .002 \\
\hline \multicolumn{2}{|c|}{$T L C$} & $8.02 \pm 1.64$ & $7.5 \pm 1.23$ & 1.081 & .286 \\
\hline \multicolumn{2}{|l|}{$P L T$} & $107.2 \pm 33.99$ & $156.94 \pm 33.27$ & 4.122 & .000 \\
\hline \multicolumn{2}{|c|}{$A L T(u / l)$} & $55.2 \pm 16.89$ & $46.0 \pm 17.95$ & 1.438 & .158 \\
\hline \multicolumn{2}{|c|}{$A S T(u / l)$} & $69.9 \pm 24.66$ & $44.52 \pm 17.95$ & 3.584 & .001 \\
\hline \multicolumn{2}{|c|}{$\operatorname{Albumin}(g / d l)$} & $3.26 \pm .268$ & $3.94 \pm .505$ & 4.090 & .000 \\
\hline \multicolumn{2}{|l|}{ INR } & $1.03 \pm .067$ & $1.02 \pm .065$ & .371 & .712 \\
\hline \multicolumn{2}{|c|}{ Total bil.(mgldl) } & $1.75 \pm .449$ & $.951 \pm .109$ & 9.597 & .000 \\
\hline \multicolumn{2}{|c|}{ Alpha FB(ng/ml) } & $19.75 \pm 4.29$ & $7.38 \pm 1.64$ & 13.825 & .000 \\
\hline \multicolumn{2}{|c|}{$T C(m g / d l)$} & $184.3 \pm 20.0$ & $184.45 \pm 16.04$ & .025 & .980 \\
\hline \multicolumn{2}{|c|}{$T G(m g / d l)$} & $140.1 \pm 14.22$ & $150.94 \pm 13.82$ & 2.159 & .037 \\
\hline \multicolumn{2}{|c|}{$L D L(m g / d l)$} & $96.1 \pm 17.02$ & $92.06 \pm 15.87$ & .694 & .492 \\
\hline \multicolumn{2}{|l|}{$D M$} & $4(40 \%)$ & $13(39.4 \%)$ & .001 & .973 \\
\hline
\end{tabular}

FIBscan = fibroscan,APRI=alt/platelets ratio index, FIB4=fibrosis index based on 4 parameters,TLC=total leucocytic count,PLT $=$ platelets count,INR=international normalization ratio ,alpha $\mathrm{FP}=$ alpha fetoprotein, $\mathrm{TC}=$ total cholesterol, $\mathrm{TG}=$ triglycerides, $\mathrm{LDL}=$ low density lipoprotein, $\mathrm{DM}=$ diabetes mellitus, bil= bilirubin,FBS=fasting blood sugar, ALT=alanine aminotransferase,AST=asprtate aminotransferase 
Table 4. Comparison between patients regarding treatment history.

\begin{tabular}{|c|c|c|c|c|c|}
\hline \multicolumn{2}{|c|}{ Variable } & Experienced $(n=7)$ & $\begin{array}{l}\text { Naïve } \\
(n=36)\end{array}$ & $t / \chi^{2}$ & $P$ \\
\hline \multicolumn{2}{|c|}{$\begin{array}{l}\text { Age (years) } \\
\text { Mean } \pm S D\end{array}$} & $50.0 \pm 10.28$ & $48.22 \pm 10.38$ & .415 & .680 \\
\hline \multirow[t]{2}{*}{ Sex } & Male & $4(57.1 \%)$ & $16(44.4 \%)$ & \multirow[t]{2}{*}{.379} & \multirow[t]{2}{*}{.538} \\
\hline & Female & $3(42.9 \%)$ & $20(55.6 \%)$ & & \\
\hline \multicolumn{2}{|c|}{ FIB scan (kpa) } & $12.9 \pm 3.73$ & $7.85 \pm 2.52$ & 4.480 & .000 \\
\hline \multicolumn{2}{|c|}{$A P P R I$} & $1.55 \pm .94$ & $.829 \pm .462$ & 3.139 & .003 \\
\hline \multicolumn{2}{|c|}{ FIB4 } & $4.42 \pm 2.39$ & $2.23 \pm .952$ & 4.176 & .000 \\
\hline \multicolumn{2}{|c|}{$F B S(m g / d l)$} & $127.43 \pm 43.28$ & $111.06 \pm 34.88$ & 1.094 & .280 \\
\hline \multicolumn{2}{|c|}{ Hemoglobin $(g m / d l)$} & $12.67 \pm 1.25$ & $13.55 \pm 1.07$ & 1.944 & .059 \\
\hline \multicolumn{2}{|c|}{$T L C$} & $8.16 \pm 1.22$ & $7.52 \pm 1.35$ & 1.166 & .250 \\
\hline \multicolumn{2}{|c|}{$P L T$} & $118.86 \pm 35.09$ & $150.53 \pm 38.37$ & 2.022 & .051 \\
\hline \multicolumn{2}{|c|}{$A L T(u / l)$} & $47.86 \pm 18.13$ & $48.19 \pm 18.17$ & .045 & .964 \\
\hline \multicolumn{2}{|c|}{$A S T(u / l)$} & $66.86 \pm 27.43$ & $47.22 \pm 19.97$ & 2.239 & .031 \\
\hline \multicolumn{2}{|c|}{$\operatorname{Albumin}(g / d l)$} & $3.66 \pm .739$ & $3.81 \pm .507$ & .678 & .502 \\
\hline \multicolumn{2}{|c|}{$I N R$} & $1.029 \pm .049$ & $1.022 \pm .068$ & .234 & .816 \\
\hline \multicolumn{2}{|c|}{ Total bil.(mgldl) } & $1.56 \pm .585$ & $1.06 \pm .32$ & 3.294 & .002 \\
\hline \multicolumn{2}{|c|}{ Alpha FB(ng/ml) } & $16.67 \pm 6.62$ & $9.01 \pm 4.84$ & 3.610 & .001 \\
\hline \multicolumn{2}{|c|}{$T C(m g / d l)$} & $188.86 \pm 20.69$ & $183.56 \pm 16.13$ & .761 & .451 \\
\hline \multicolumn{2}{|c|}{$T G(m g / d l)$} & $143.29 \pm 15.96$ & $149.42 \pm 14.23$ & 1.024 & .312 \\
\hline \multicolumn{2}{|c|}{$L D L(m g / d l)$} & $88.71 \pm 16.18$ & $93.83 \pm 16.09$ & .769 & .446 \\
\hline \multicolumn{2}{|c|}{$D M$} & $4(57.1 \%)$ & $13(36.1 \%)$ & 1.060 & .303 \\
\hline
\end{tabular}

FIBscan $=$ fibroscan,APRI=alt/platelets ratio index, FIB4=fibrosis index based on 4 parameters,TLC=total leucocytic count,PLT $=$ platelets count,INR=international normalization ratio ,alpha $\mathrm{FP}=$ alpha fetoprotein, $\mathrm{TC}=$ total cholesterol, $\mathrm{TG}=$ triglycerides, $\mathrm{LDL}=\mathrm{low} \quad$ density lipoprotein,DM= diabetes mellitus,FBS=fasting blood sugar.total bil=total bilirubin , ALT=alanine aminotransferase,AST=asprtate aminotransferase 
Table 5. Multivariate regression analysis to identify factors associated with failure of liver stiffness improving.

\begin{tabular}{|c|c|c|c|c|c|c|}
\hline & \multirow[t]{2}{*}{$\beta$} & \multirow[t]{2}{*}{ S.E. } & \multirow[t]{2}{*}{ Sig. } & \multirow[t]{2}{*}{$O R$} & \multicolumn{2}{|c|}{$95 \%$ CI for $O R$} \\
\hline & & & & & Lower & Upper \\
\hline Age & .130 & .177 & .463 & 1.138 & .805 & 1.609 \\
\hline $\operatorname{Sex}(F$ vs $M)$ & 1.403 & .932 & .132 & 0.246 & .040 & 1.529 \\
\hline$D M$ & 1.406 & 1.691 & .406 & 4.080 & .148 & 2.187 \\
\hline T. Bil(mg/dl) & .939 & 1.171 & .423 & 2.557 & .258 & 5.362 \\
\hline$I N R$ & .797 & .720 & .104 & 6.403 & .563 & 7.912 \\
\hline APRI & .294 & .461 & .001 & 12.461 & .641 & 6.348 \\
\hline FIB4 & .649 & .261 & .000 & 9.164 & .265 & 8.649 \\
\hline Alpha FB(ng/ml) & .164 & .346 & .007 & 4.316 & .894 & 2.491 \\
\hline FIB SCAN & .023 & .330 & .038 & 1.024 & .536 & 1.954 \\
\hline$S V R$ & 4.294 & 1.664 & .000 & $14 . .26$ & 2.35 & 32.164 \\
\hline
\end{tabular}

FIBscan $=$ fibroscan,APRI=alt/platelets ratio index, FIB4=fibrosis index based on 4 parameters,TLC=total leucocytic count,PLT= platelets count,INR=international normalization ratio , alpha $\mathrm{FP}=$ alpha fetoprotein, ,DM= diabetes mellitus.,SVR=sustained virological response.F=female,M=male,T.BIL=total bilirubin,

\section{DISCUSSION}

The Egyptian health authorities provided direct antiviral agents( DAAs) regimens as an a treatment for patients infected with $\mathrm{HCV}$ at 2014 [9].

This treatment can achieve SVR, DAAs do not have an anti-fibrotic impact however primarily involved with viral eradication [10].

This results in resolution of the hepatic damage, and decline hepatic cell failure complications like variceal hemorrhage and liver cancer [11].

$\mathrm{HCV}$ can be a great factor of liver fibrosis and an important agent of liver injury and inflammation [12].
In spite of the gradual decrease in liver fibrosis once SVR, hepatic pathology is still its accurate and repeated estimation is required [13].

The aim of the present study was to assess the changes in liver stiffness measurements by transient elastography and also the variations in indices of fibrosis as determined by APRI, FIB4 following $\mathrm{HCV}$ treatment with sofosbuvir based regimens. Estimating its effect without IFN on the changes of liver fibrosis measuring by fibroscan device and hepatic fibrosis indices was done.

Similar to Bachofner, et al. [13], and Elsharkawy, et al. [14] who found that LS measurements considerably declined twelve weeks following the end of antiviral therapy, 
our study revealed that LS considerably declined 48weeks following the end of antiviral drug treatment in patients who achieve SVR. In addition, there was a significant improvement in APRI and FIB4 scores. These scores are affected by the declining of liver enzymes and platelets count denoting vital improvement of liver pathology and necro inflammation following Sofosbuvir based treatment. Also, treatment responders showed vital decrease in these indices whereas decline of these indices in relapsers wasn't obvious [14].

Our results showed significant improvement in hepatic fibrosis measuring in responders patients $(90.6 \%)$ while in non-responders patients $(9.4 \%)$ no significant decrease in LS measurements. In cirrhotic patients (23.2\%) receive the treatment there was significant improvement of LS measuring, 50\% of these patients became non cirrhotic and also the alternative $50 \%$ persisted but with degrees of liver fibrosis less than degrees before treatment may be due to persistent vireamia and treatment faiure and these results are in agreement with Elsharkawy et al. showed significant improvement in hepatic fibrosis measuring in $81.1 \%$ of cirrhotic patients, but they stay to be cirrhotic with liver fibrosis degrees less than what before treatment [13]. D'ambrosio et al, found there is cirrhosis even in patients who achieve sustained virological response (SVR) [15]. Chekuri et al reported important decline in hepatic fibrosis degree in patients who are cirrhotic, but sixty percent who had cirrhotic before treatment were still cirrhotic at SVR24 [12].

\section{CONCLUSION}

Drug regimens containing sofosbuvir make a major decline in liver pathology degree proved by utilizing the fibroscan and fibrosis indices like APRI and FIB4 test even in cirrhotic patients, but starting treatment early is imperative except liver injury will be constant . These non-invasive fibrosis scores might be useful in the developing regions of restricted resources for patients follow up. Long term keeping eye on these patients is recommended to completely perceive the effect of SVR on liver pathology dynamics.

\section{Declaration of interest}

The authors report no conflicts of interest. The authors alone are responsible for the content and writing of the paper.

\section{Funding information}

None declared

\section{REFERENCES}

1) Yousra A , Ghina R, Riome S, Miller D and Laith $\mathrm{J}$ : the epidimology of hepatitis $\mathrm{C}$ virus in Egypt :a systemic review and data synthesis, BMC Infect Dis 2013.

2) Lawitz E, Poordad FF, Pang PS, Hyland RH, Ding X, Mo $\mathrm{H}$ et al. Sofosbuvir and ledipasvir fixed-dose combination with and without ribavirin in treatment-nai"ve and previously treated patients with genotype 1 hepatitis $\mathrm{C}$ : the LONESTAR study. Hepatol 2013; 58:1092A.

3) Nyberg LM LX, Yang S , Chiang, Cheetham CT , Caparosa S, Pio J et al. The association of sustained virological response and all-cause mortality after interferon-based therapy for chronic hepatitis $\mathrm{C}(\mathrm{HCV})$ in a large U.S community-based health care delivery system. Oral presentation at: American Association for the study of liver diseases.2015.

4) Ziol M, Handra-Luca A , Kettaneh A , Christidis C, Mal F, Kazemi $F$ et al. Noninvasive assessment of liver fibrosis by measurement of stiffness in patients with chronic hepatitis C. Hepatol 2005; 41: 48-5

5) Sandrin L, Oudry J , Bastard C, Fournier C, Miette $\mathrm{V}$ and Muller $\mathrm{S}$ : Non-Invasive Assessment of Liver Fibrosis by VibrationControlled Transient Elastography (Fibroscan®).2011

6) Talwalkar JA, Kurtz DM, Schoenleber SJ, West $\mathrm{CP}$ and Montori VM. Ultrasound-based transient elastography for the detection of hepatic fibrosis: systematic review and meta-analysis. Clin Gastroenterol Hepatol 2007; 5: 1214-1220.

7) Karlas T, Benckert J, Beer S, Keim V, Berg T and Wiegand $\mathrm{J}$ : can persisting liver stiffness indicate increased risk of HCC, after successful anti-HCV therapy? Aliment Pharmacol Ther .2016

8) Wai CT, Greenson JK, Fontana RJ, Kalbfleisch JD, Marrero LA, Conjeevaram HS et al. A simple noninvasive index can predict both significant fibrosis and cirrhosis in patients with chronic hepatitis C. Hepatol 2003; 38: 518-26. 
9) EASL (2017). Recommendations on Treatment of Hepatitis C. J Hepatol 2016; 66:153-194.

10) George SL, Bacon BR, Brunt EM, Mihindukula suriya KL, Hoffmann $\mathrm{J}$ and Di Bisceglie AM.: Clinical , virologic ,histologic biochemical outcomes after successful HCV therapy: a 5-year follow-up of 150 patients. Hepatol 2009; 49: 729-38.

11) Marcellin $P$, Boyer $N$, Gervais $A$, Martinot $M$, Pouteau M, Castelnau C et al. Long-term histologic improvement and loss of detectable intrahepatic HCV RNA in patients with chronic hepatitis $\mathrm{C}$ and sustained response to interferon alpha therapy. Ann Intern Med1997; 127: 87581.

12) Chekuri S, Nickerson J, Bichoupan $K$, Sefcik R , Doobay K , Chang S , et al. Liver Stiffness Decreases Rapidly in Response to Successful Hepatitis C Treatment and Then Plateaus. PLoS ONE 2016; 11: e0159413.
13) Elsharkawy A, Abdel Alem S, Fouad R, El Raziky M , El Akel W, Abdo M, et al. Changes in Liver stiffness measurements and Fibrosis scores following Sofosbuvir based treatment regimens without Interferon. J Gastroenterol Hepatol 2017; 32: 1624-1630.

14) Bachofner JA, Valli PV, Kröger A, Bergamin I, Künzler P, Baserga A, et al. Direct antiviral agent treatment of chronic hepatitis $\mathrm{C}$ results in rapid regression of transient elastography and $\mathrm{fi}$ brosis markers fi brosis-4 score and aspartate aminotransferase-platelet ratio index. Liver Int. 2017; 37: 369-376.

15) doi: 10.1111/liv.13256. Epub 2016 Nov 3.

16) D'Ambrosio R, Aghemo A, Rumi MG, Ronchi G , Donato MF, Paradis'V , et al. A morphometric and immunohistochemical study to assess the benefit of a sustained virological response response in hepatitis $\mathrm{C}$ virus patients with cirrhosis. Hepatology 2012; 56(2):532-43.

To Cite This Article: MounirMH, Ibrahim AM, BayiomySS, Mohammed HM. Assessment of Liver Stiffness among Chronic Hepatitis C Patients after Oral Antiviral Drugs in Zagazig University Hospitals. ZUMJ 2019; 25 (5): 630-638. Doi: 10.21608/zumj.2019.11090.11450. 